\title{
What happens on Cygnus X-3?
}

\section{The notion that a periodic X-ray source may also be giving off streams of massive particles which are unknown from accelerator experiments is still, against the odds, alive.}

Outwardey, Cygnus X-3 is a run-of-the mill celestial source of $X$-rays, first recognized as such (in 1966) by early rocket observations which have since been amply confirmed by measurements from Earth satellites. One of the most striking features of the object is that it is a regularly periodic source of $\mathrm{X}$ rays with a period of 4.8 hours, which fits in well with the assumption that it is a binary star, one partner of which is a massive neutron star while the other is a less compact, more "normal", companion.

Cygnus X-3 is also distinguished by its intensity as a source of $X$ rays. Indeed, the source is thought to be rather more than 10,000 parsecs distant from the Solar System, a substantial fraction of the diameter of the Galaxy. But this circumstance goes only to sharpen another puzzling feature of the properties of the object, that it is a source not merely or ordinary $\mathrm{X}$ rays but of $\mathrm{X}$ rays so energetic that they are properly known as gamma rays. Indeed, there have been suggestions (see, for example, A.M. Hillas, Nature 312,$50 ; 1984)$ that a few sources as powerful as Cygnus X-3 might be sufficient to account for the total content of cosmic rays in the Galaxy.

This leads to the most curious and unexpected feature of Cygnus X-3, the sketchy but intriguing suggestion that this very distant binary star may be the source of energetic particles whose nature is at present entirely unknown but which are nevertheless responsible for the production of mu-mesons (muons) at the surface of the Earth. The evidence is sketchy in the sense that it is urgently in need of independent verification, but it is also the kind of evidence that people cannot entirely set to one side.

Observations that suggest some connection between the X-ray star and the detection of cosmic rays at the surface of the Earth go back for several years. In 1983, for example, J. Lloyd-Evans and a group of colleagues at the University of Leeds (see Nature 305, 705; 1983) showed that there is a correlation between the detection of energetic cosmic-ray showers in the atmosphere of the Earth and the phase of the supposed binary source of $\mathrm{X}$ rays from Cygnus $\mathrm{X}-3$. Whatever else may emerge about this strange object, two things are clear from the cosmic-ray observations: first, the object is a source of energetic cosmic rays that are detectable at the surface of the Earth; and, second, that the sheer production of energy in the form of energetic particles, say gamma-ray photons, must be very large.

The measurements that have raised new possibilities were undertaken with the equipment installed deep underground in recent years in the hope of detecting the predicted radioactive decay of the proton. An unavoidable complication of these measurements is the background flux of energetic muons, which explains why there have recently accumulated data on the occurrence near the surface of the Earth of muons energetic enough to penetrate at least through the overburden of rock to the subterranean detectors.

The first reports that something remarkable may be afoot appeared just over a year ago (M.L. Marshak et al., Phys. Rev. Lett. 54, 2079; 1985), and were based on measurements of background muons at the proton-decay detector operated jointly by the University of Minnesota and the Argonne National Laboratory. What Marshak and his colleagues claimed is that muons observed deep underground correlate significantly with the phase of the distant X-ray star in its binary orbit. Supporting evidence has been produced by $\mathrm{G}$. Battistoni et al., based on measurements with the proton-decay equipment under Mont Blanc (Phys. Lett. 155B, 465; 1985).

For the past year, the bare bones of the mystery of Cygnus X-3 have been plain for all to see. If there is indeed a correlation between the phase of Cygnus X-3 and the time of detection of energetic cosmic-ray particles of some kind at the surface of the Earth, the implication is that the propagation of some influence over the 10,000 parsecs between the double source and the Solar System must be accomplished in such a way that phase information is not lost on the journey, which in turn implies that differences of travel time over 10,000 parsecs must be a small fraction of the orbital period, a few minutes perhaps.

Since these circumstances were first appreciated, there has been a gale of speculation about the means by which the influence of Cygnus X-3 might be propagated over these distances. First, the precision of the timing implies that the speed of propagation must be a very large proportion of the velocity of light, which in turn implies that if particles are the medium by which the message is transmitted, they cannot be massive particles. Indeed, Marshak et al. last year gave good reasons why particles as massive as neutrons would not meet the need, while particles as light as photons were similarly excluded on the grounds that there would have to be more of them than could possibly be consistent with other observations.

K. Ruddick (from the Minnesota group) now takes the argument further by pursuing the suggestion, originally made by Marshak et al., that the intermediary between Cygnus X-3 and the Solar System is a flux of particles that are electrically neutral (so as not to be smeared out by the intragalactic magnetic field), relatively small in mass (so as to preserve the synchroneity of the events) and previously unknown. Ruddick's account of the problem appears in Phys. Rev. Lett. 57, 531; 1986. The essence of his case is that light neutral particles are transmitted from Cygnus X-3 until they meet the rocks of the surface of the Earth, whereupon they are converted into other particles.

In principle, the argument is simple enough. Here is a set of data that cannot easily be explained in terms of existing theories. so why not invent a novel particle to account for what may be happening? The innovation in Ruddick's argument is that the primary novel particles (called "cygnets", which is natural enough given where they come from) do not interact directly with terrestrial nucleons to give the muons observed, but instead do so through the intermediary of a second unknown particle. necessarily more massive than the first. Ruddick argues that neither particle need have been observed in accelerator experiments.

The temptation to mock at the inven ion of a new particle of matter to solve each new problem in astrophysics should be firmly suppressed. By any yardstick. Cygnus X-3 is a remarkable object. Most of what people have written about it has been concerned with explaining how it may function as a source of very energetic $X$ rays. Several models have been ad anced, most of which entail the impact of a particle beam from the neutron star on the more extended envelope of its larger companion. Until the likely spectral distribution of the energy produced by such a process is more exactly known, it will be hard to tell how strong is the case for extra particles. And, meanwhile, there is a great need that the original measurements should be repeated in such a way that people can tell more precisely how critical is the apparent synchroneity of phase. 